International Journal of Pure and Applied Mathematics

Volume 86 No. 4 2013, 669-688

ISSN: 1311-8080 (printed version); ISSN: 1314-3395 (on-line version)

url: http://www.ijpam.eu

doi: http://dx.doi.org/10.12732/ijpam.v86i4.7

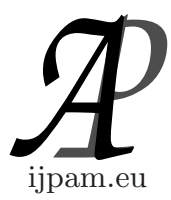

\title{
VERTEX COLORING OF CERTAIN DISTANCE GRAPHS
}

\author{
V. Yegnanarayanan ${ }^{1} \S$, A. Parthiban ${ }^{2}$ \\ ${ }^{1,2}$ Department of Mathematics \\ Velammal Engineering College \\ Ambattur-Redhills Road, Chennai, 600 066, Tamil Nadu, INDIA
}

\begin{abstract}
In this paper first, we give a brief introduction about integer distance graphs. An integer distance graph is a graph $G(Z, D)$ with the set of integers as vertex set and an edge joining two vertices $u$ and $v$ if and only if $|u-v| \in D$ where $D$ is a subset of the positive integers. If $D$ is a subset of $P$ then we call $G(Z, D)$ a prime distance graph. Second, we obtain a partial solution to a general open problem of characterizing a class of prime distance graphs. Third, we compute the vertex arboricity of certain prime distance graphs. Fourth, we give a brief review regarding circulant graphs and highlight its importance in the computation of chromatic number of distance graphs with appropriate references. Fifth, we introduce the notion of pseudochromatic coloring and obtain certain results concerning circulant graphs and distance graphs.
\end{abstract}

AMS Subject Classification: $05 \mathrm{C} 15$

Key Words: chromatic number, circulant graphs, coloring, prime distance graphs

\section{Introduction}

Let $G$ be a graph. A vertex coloring of $G$ is an assignment of colors to its vertices so that no two adjacent vertices receive the same color. The chromatic number $\chi(G)$ is the minimum number of colors needed to color $G$. An equivalent

Received: April 1, 2013

(C) 2013 Academic Publications, Ltd.

$\S$ Correspondence author url: www.acadpubl.eu 
definition of the chromatic number is the minimum integer $k$ such that there is a partition of the vertex set of a given graph into $k$ sets so that the subgraph induced by each set is an independent set.

The distance graph, first introduced by Eggleton, Erdos and Skilton [25], is motivated by the well-known Hadwiger-Nelson problem which asks for the minimum number of colors needed to color all points of the plane such that points at unit distances receive different colors. Motivated by the plane coloring problem, one can consider the analogue to the one-dimensional case by investigating the chromatic numbers of distance graphs on the real line $R$ and the integer set $Z$.

If $D$ is a subset of the set of positive integers, then the integer distance graph $G(Z, D)$ is defined to be the graph with vertex set $Z$, where two vertices $u$ and $v$ are adjacent if and only if $|u-v| \in D$. A particularly interesting problem is determining the chromatic number of $G(Z, D)$ for a given set $D$. The chromatic number of integer distance graphs, denoted $\chi(G(Z, D))$ has been studied extensively in [7], [49], [50], [51], [63] for different families of distance sets $D$. Thus, $D$ is the set of forbidden distances with respect to coloring the integers on the real line.

In fact much work has been done on this problem in [6], [8], [9], [25], [26], [34], [35], [37], [38], [39], [40], [41], [42], [46], [49], [50], [51].

\section{Prime Distance Graphs}

Let $P$ denote the set of all primes, and let $D \subseteq P$ be a prime set. As $D$ does not contain any multiples of 4 , the modular 4 function on integers gives a proper coloring for $G(Z, D)$. Eggleton et al. in [22], [23], [24], [25], [26] proved 1) $\chi(G(Z, P))=4$. 2) For 3-element prime sets $D, \chi(G(Z, D))=4$ if and only if $D=\{2,3,5\}$. 3) If $2 \notin D$, then $\chi(G(Z, D))=2$. 4) If $3 \notin D$, then $\chi(G(Z, D)) \leq 3$, and equality holds if and only if $2 \in D$ and $|D| \geq 2$. Hence, to study the chromatic number of a distance graph generated by a prime set it bounds to finding the chromatic number of $D$ with $\{2,3\} \in D$, for which $\chi(G(Z, D))$ is either 3 or 4 . In the case of former it is usually called a class 3 set and in the latter, it is called a class 4 set. Eggleton et al. also proved in [22], [23], [24], [25], [26] that 5) if $D \subseteq P$ and $D=\{2,3, p, p+2\}$ with $p$ and $p+2$ as twin primes, then $D$ is a class 4 set. Eggleton et al. [24] gave the following conjecture:

Conjecture 2.1. Let $D$ be a subset of the set $P(\{2,3\}) \subseteq D)$ of primes. Then $\chi(D)=4$ if and only if $D$ contains a twin of primes. 
It is easy to see that $\chi(G(Z, D))=4$ if $D$ contains a twin of primes. The second part of this conjecture is disproved in [23] and in [52] by counterexamples. M. Voigt and H. Walther proved the Theorem 2.1 in [50]. For more on the computation of chromatic number of prime distance graphs refer [54], [55], [56], [57], [58], [59], [60], and [61]. Moreover, Voigt and Walther [50], [51] completely characterized all 4-element class 4 prime sets as follows.

Theorem 2.1. Let $D=\left\{2,3, p_{1}, p_{2}\right\}$ be a set of primes with $p_{1} \geq 7$ and $p_{2}>p_{1}+2$. Then $\chi(G(Z, D))=4$ holds if and only if $\left(p_{1}, p_{2}\right) \in$ $\{(11,19),(11,23),(11,37),(11,41),(17,29),(23,31),(23,41),(29,37)\}$.

For a set $D$ of positive integers, a sequence $S$ of non-negative integers is called a $D$-sequence if $|x-y| \notin D$ for any $x, y \in S$. Let $S(n)=\mid S \cap\{0,1,2, \ldots, n-$ $1\} \mid$. The density of $S$, denoted $\delta(S)$, is defined by $\delta(S)=\lim _{n \rightarrow \infty} \frac{s(n)}{n}$.

The parameter of interest is the density of $D, \mu(D)$, defined by

$$
\mu(D)=\sup \{\delta(S): S \text { is a } D \text {-sequence }\} .
$$

In 1975, Cantor and Gordon [5] proved the existence of $\mu(D)$ for any $D$. The exact values of $\mu(D)$ for several families of sets $D$ were studied by Haralambis [30]. The parameter $\mu(D)$ is also closely related to the channel assignment problem (or T-coloring). Griggs and Liu [29] indicated that $\mu(D)$ is equivalent to the asymptotic ratio of $T$-colorings for cliques and gave a different proof for the existence of $\mu(D)$, using directed graphs.

For a real number $x$, let $\|x\|$ denote the distance from $x$ to the nearest integer, i.e.,

$$
\|x\|=\min \{x-\lfloor x\rfloor,\lceil x\rceil-x\} .
$$

For a set $D \subset R$ and $t \in R$, let $\|t D\|=\inf \{\|t x\|: x \in D\}$, and define $\kappa(D)=\sup \{\|t D\|: t \in R\}$. The parameter $\kappa(D)$ is studied in the Diophantine approximations by Wills [53], Y.G. Chen [10], [11], [12], the View Obstruction Problems by the authors in [17], [18], [19] and Cusick and Pomerance [20], and problems concerning flows and colorings of graphs by Bienia et al. [4].

Now we consider the problem of characterizing distance sets with cardinality 5. That is, if $D=\left\{2,3, p_{1}, p_{2}, p_{3}\right\} \subseteq P$ then what is $\chi(G(Z, D))$ ?

Theorem 2.2. Let $D=\left\{2,3, p_{1}, p_{2}, p_{3}\right\} \subseteq P$ with $p_{1} \equiv 1$ or $2(\bmod 3)$, $p_{2}=p_{1}+10$ and $p_{3}>p_{2}$. Then $\chi(G(Z, D))=3$.

Proof. For suitable primes $p_{1}, p_{2}$, and $p_{3}$ we proceed as follows: For a set $D$, let $\alpha, r \leq 2 \max D$ be positive integers, with $\operatorname{gcd}(\alpha, r)=1$. Let $|\alpha D|_{r}=$ $\min \left\{|\alpha d|_{r}: d \in D\right\}$, where $|x|_{r} \equiv x(\bmod r)$ and $\eta_{1}(D)=\max \left\{|\alpha D|_{r} \mid r: 1 \leq\right.$ 
$r, \alpha \leq 2 \max D, \operatorname{gcd}(\alpha, r)=1\}$. If $p_{1} \equiv 1(\bmod 3)$, then let $m=2 p_{1}+10$. Clearly $m \equiv 12(\bmod 6)$. So $m / 3 \equiv 4(\bmod 2)$. Hence it is sufficient to find $\alpha$ such that for all $d \in D, k_{1} \leq|\alpha d|_{m} \leq 2 k_{1}$ where $k_{1} \equiv 4(\bmod 2) \ldots$ (1) Let $B \equiv\left[k_{1} / 2, k_{1} / 2+\left\lfloor k_{1} / 6\right\rfloor\right]$. Then $\left|2 x_{1}\right|_{m}$ and $\left|3 x_{1}\right|_{m}$ satisfy $(1)$, for any $x_{1} \in B$. It is easy to note that $k_{1} / 2,\left(k_{1} / 2\right)+1 \in B$. Now let $\alpha=\left(k_{1} / 2\right)+1$ or $k_{1} / 2$. Then it follows that $\left|\alpha p_{1}\right|_{m}$ satisfies (1). Next if $p_{1} \equiv 2(\bmod 3)$, then let $m=2 p_{1}+10$. Clearly $m=14(\bmod 6)$. So $\lceil m / 3\rceil \equiv 5(\bmod 2)$. Hence it is sufficient to find $\alpha$ such that $k_{1} \leq|\alpha d|_{m} \leq 2 k_{1}-1$, where $k_{1} \equiv 5(\bmod 2)$...(2). Let $B=\left[\left\lceil k_{1} / 2\right\rceil,\left\lceil k_{1} / 2\right\rceil+\left\lfloor k_{2} / 3\right\rfloor\right]$, where $k_{1}=2 k_{2}+5$. Then $\left|2 x_{1}\right|_{m}$ and $\left|3 x_{1}\right|_{m}$ satisfy (2), for any $x \in B$. Now let $\alpha=\left\lceil k_{1} / 2\right\rceil$ or $\left\lceil k_{1} / 2\right\rceil+1$. Then it follows that $\left|\alpha p_{1}\right|_{m}$ satisfies (2). It is easy to see that $\eta_{1}(D) \geq 1 / 3$. Chang et al. in [7] and Zhu in [64] have shown that $\frac{1}{\mu(D)} \leq \chi(G(Z, D)) \leq\left\lceil\frac{1}{\kappa(D)}\right\rceil$. When $D$ is a finite set then it follows immediately that $\kappa(D)=\eta_{1}(D)$. So $\chi(G(Z, D)) \leq 3$. It is a simple exercise to exhibit a chromatic 3-coloring for $G(Z, D)$.

As a generalization of Theorem 2.2 one can have the following result.

Theorem 2.3. Let $D=\{2,3\} \cup\left\{p_{i}: p_{i} \in P\right.$, with $p_{1} \equiv 1$ or $2(\bmod 3)$, $p_{2}=p_{1}+10$ and $p_{i}>p_{2}$ for $\left.i=3,4, \ldots\right\}$. Then $\chi(G(Z, D))=3$.

Proof. On similar lines as in Theorem 2.2, as $D=\left\{2,3, p_{1}, p_{2}, p_{3}\right\}$ of Theorem 2.2 is a subset of $D=\{2,3\} \cup\left\{p_{i}: p_{i} \in P\right.$, with $p_{1} \equiv 1$ or $2(\bmod 3)$, $p_{2}=p_{1}+10$ and $p_{i}>p_{2}$ for $\left.i=3,4, \ldots\right\}$ and $\chi$ is a monotonically increasing function.

Open Problem 2.1. Characterize completely the class 3 and Class 4 sets $D$ of any cardinality.

It is a well known conjecture that there are arbitrarily long arithmetic progressions of prime numbers. The conjecture is best described as "classical". In Diekson's History it is stated that around 1770 Lagrange and Waring investigated how large the common difference of an arithmetic progression of $L$ primes must be and it is hard to imagine that they did not at least wonder whether their results were sharp for all $L$.

It is not surprising that the conjecture should have been made, since a simple heuristic based on the prime number theorem would suggest that there are $\gg \frac{N^{2}}{\log ^{k} N} k$-tuples of primes $p_{1}, p_{2}, \ldots, p_{k}$ in arithmetic progression, each $p_{i}$ being at most $N$. Hardy and Littlewood [31] in their famous paper of 1923, advanced a very general conjecture which, as a special case, contains the hypothesis that the number of such $k$-term progressions is symtotically $\frac{C_{k} N^{2}}{\log ^{k} N}$ for a certain explicit numerical factor $C_{k}>0$. Green and Tao in [2] do not come close to establishing this conjecture but instead gave a lower bound $(\gamma(k)+o(1)) \frac{N^{2}}{\log ^{k} N}$ for some very 
small $\gamma(k)>0$.

The first theoretical progress on these conjectures was made by Van der corput [48] who in 1939, used Vinogradov's method of prime number sums to establish the case $k=3$. That is, there are infinitely many triplets of primes in arithmetic progression. However, the question of longer arithmetic progression seems to have remained completely open even for $k=4$. On the other hand, it has been known for some time that better results can be obtained if one replaces the primes with a slightly larger set of at most primes. The most impressive such result is due to Heath-Brown [32]. He showed that there are infinitely many 4term progressions consisting of three primes and a member which is either prime or a product of two primes. The problem of finding long arithmetic progressions in the primes has also attracted the interest of computational mathematicians. According to Green-Tao [2] the longest known arithmetic progressions of primes is of length 23, and was found in 2004 by Markus Fruid, Paul underwood and Paul Jobling. Finally Green and Tao [2] settled the conjecture concluding: "The prime numbers contain infinitely many arithmetic progressions of length $k$ for all $k . "$

In view of the above discussion on the arithmetic progression of primes it is easy to deduce that there are infinitely many finite (or infinite) prime distance sets with chromatic number 3 or 4 .

\section{Vertex Arboricity of Prime Distance Graphs}

A $k$-coloring of a graph $G$ is a mapping $f$ from $V(G)$ to $\{1,2, \ldots, k\}$. With respect to a given $k$-coloring, let $V_{i}$ denote the set of all vertices of $G$ colored with $i$, and $\left\langle V_{i}\right\rangle$ denotes the subgraph induced by $\left\langle V_{i}\right\rangle$ in $G$. If $\left\langle V_{i}\right\rangle$ is an independent set for every $1 \leq i \leq k$, then $f$ is called a proper $k$-coloring. The chromatic number of a graph $G$ is the minimum number of $k$-color for which $G$ has a proper $k$-coloring. If $\left\langle V_{i}\right\rangle$ induces a subgraph whose connected components are paths, then $f$ is called a path $k$-coloring. The vertex arboricity of a graph $G$, denoted by $a(G)$ is the minimum number of $k$ for which $G$ has a path $k$-coloring.For any $D \subseteq P$ where the set $P$ is the set of all prime numbers, we call $G(Z, D)$ a prime distance graph. If $|D| \leq 1$ then $a(G(Z, D))=1$ since the graph consists of paths only. Suppose that $|D|=2$ and let $D=\{p, q\} \subset P$ with $p<q$. Now set $g(0)=g(1)=\ldots=g(q-1)=1, g(q)=g(q+1)=\ldots=g(2 q-1)=2$, and $g(x+2 q)=g(x)$ for all $x \in Z$. Then $g$ is a path coloring and $a(G(Z, D)) \leq 2$. As we find a cycle $0, p, 2 p, \ldots, q p,(p-1) q, \ldots, 2 q, q, 0$ in this graph, we deduce that $a(G(Z, D))=2$. If $D$ is a finite set, then $G(Z, D)$ is $2|D|$-regular graph 
and $a(G(Z, D)) \leq|D|$.

Theorem 3.1. For any prime $p$ and $k>1$, let $D^{*}=\{p+1, p+2, \ldots, p+k\}$ be a set of integers. Then $a\left(G\left(Z, D^{*}\right)\right) \leq\lceil(p+k) /(p+3)\rceil+1$.

Proof. Let $s=\lceil(p+k) /(p+3)\rceil$, with $g(y(p+3)+j)=y$ for $0 \leq y \leq s$ and $0 \leq j \leq p+2$, and $g((s+1)(p+3)+x)=g(x)$ for all $n \in Z$. Then $g$ is a path coloring. Hence $a\left(G\left(Z, D^{*}\right)\right) \leq s+1=\lceil(p+k) /(p+3)\rceil+1$.

Theorem 3.2. If $D=\{p, 2 p, \ldots, n p, q\}$ where $p, q$ are primes then

$$
a(G(Z, D))=\lceil(n+1) / 2\rceil=\lceil|D| / 2\rceil .
$$

Proof. Since $D_{1}=\{p, 2 p, \ldots, n p\} \subset D$ and $a\left(G\left(Z, D_{1}\right)\right)=\lceil(n+1) / 2\rceil$, $a(G(Z, D)) \geq\lceil(n+1) / 2\rceil=\lceil|D| / 2\rceil$. To prove the converse, suppose that $n$ is odd. A coloring $g$ of $G(Z, D)$ is called $s$-admissible if $g(v) \neq g(v+s)$ for all $v \in Z$, and $S$-admissible if it is $s$-admissible for all $s \in S$. A coloring $g$ is called $y$-periodic if $g(v)=g(v+y)$ for all $v \in Z$. We establish that $a(G(Z, D)) \leq\lceil|D| / 2\rceil$ by building a $(n+1) p$-periodic path coloring. As $q \neq 0$ $(\bmod (n+1) p)$ we can presume without loss of generality that $0<q<(n+1) p$. The periodic coloring defined by unlimited repetition of the below mentioned coloring section of length $(n+1) p$ is $z$-admissible for $z$ with $2 p \leq z \leq n p$, since vertices of same color are of distance less than $2 p$ or greater than $n p$.

$$
\begin{aligned}
T_{(n+1) p} & =(0)^{2 p}(1)^{2 p} \ldots\left(\frac{n-3}{2}\right)^{2 p}\left(\frac{n-1}{2}\right)^{2 p} \\
& =(0, \ldots 0)(1, \ldots, 1), \ldots\left(\frac{n-1}{2}, \ldots, \frac{n-1}{2}\right)
\end{aligned}
$$

Hence this coloring is $\{2 p, \ldots, n p\}$-admissible as well as $q$-admissible if $2 p \leq$ $q \leq n p$. Now we have to consider the cases that $0<q<2 p$ and $n p<q<$ $(n+1) p$. Since any $(n+1) p$-periodic $q$-admissible coloring with $0<q<2 p$ is also admissible for $q^{\prime}=(n+1) p-q$ with $n p<q^{\prime}<(n+1) p$, it only remains to consider the case that $0<q<2 p$. Let $2 p=w q+w_{1}, w \in N, w_{1} \in Z$ with $0 \leq w_{1}<q$. Defining coloring sections of length $2 p$ by

$$
T_{2 p}^{(g, g+2)}(q)= \begin{cases}(g)^{q}(g+2)^{q} \ldots(g)^{q}(g+2)^{q}(g)^{w_{1}}, & \text { if } w \text { is even; } \\ (g)^{q}(g+2)^{q} \ldots(g)^{q}(g+2)^{q}(g+2)^{w_{1}}, & \text { if } w \text { is odd }\end{cases}
$$

Now build a periodic coloring of $G(Z, D)$ by fixing together $(n+1) / 2$ of such sections as given below. 


$$
T_{(n+1) p}
$$

$$
=T_{2 p}^{(0,2)}(q) \ldots T_{2 p}^{(f, f+2)}(q) \ldots T_{2 p}^{((n-3) / 2,0)}(q) T_{2 p}^{((n-1) / 2,1)}(q) .
$$

This coloring is $\{2 p, 3 p,, . ., n p\}$-admissible as the vertices $v, v+2 p, \ldots, v+n p$ are colored differently for all $v \in Z$. The periodic coloring is also $q$-admissible as the vertices of same color in a section $T_{2 p}^{(f, f+2)}(q)$ are of distance unequal to $q$ and vertices of same color in different sections are of distance greater than $y$ as $n \geq 2$. So in this coloring of $G(Z, D)$ if $v_{1}, v_{2}$ are assigned same color, then $v_{1}$ and $v_{2}$ are not adjacent or $\left|v_{1}-v_{2}\right|=p$. So the adjacent vertices of $v_{1}$ are not at most two vertices $v_{1}-p, v_{1}+p$ with the same color of $v_{1}$. However $v_{1}-p, v_{1}+p$ cannot have the same color with $v_{1}$ at the same time. So this coloring is a path coloring of $G(Z, D)$ and $a(G(Z, D)) \leq\lceil|D| / 2\rceil$.

\section{Circulant Graphs}

The analysis and design of interconnection networks is motivated by recent developments in technologies such as optical fibre and by progress in parallel and distributed computing. Engineers and scientists often use graphs to model the topological structure of an interconnection network. Switches, processing elements or memory modules correspond to the vertices of the graph while communication links corresponds to the edges. Graph theory is an efficient tool for solutions to problems often encountered in networks. Circulant graphs are used to model interconnection networks.

The design of an interconnection network should conform to the basic features such as small and fixed degree of each node in the network, small transmission cost, maximum fault tolerance, easy routing algorithms, embeddability of other topologies,symmetry, extendability, and efficient layout of VLSI circuits. Due to the cost and engineering limitations, the maximum node degree is a primary constraint in network design. Most of the popular network topologies have the drawback that the size of the network cannot be increased incrementally. Instead, the size is limited to a multiple of some factor and the number of connections to each node is proportional to the growth of the network. However the circulant graphs, a family of cayley graphs, allow for incremental extendability, with the number of connections to each node and the diameter remaining constant in the networks they model. Cayley graphs are often suggested as models for interconnection networks because of their high symmetry. Since these graphs are vertex transitive they allow for the development of efficient routing algorithms. in the case of prime number of vertices, circulants 
are the only vertex transitive graphs. For these reasons, circulant graphs have received a lot of attention.In recent years research regarding circulant graphs has expanded to areas such as recognition, hardness, spectral properties, isomorphism, enumeration, hamiltonicity and so on. It has been conjectured that every connected cayley graph on an abelian group admits hamiltonian decomposition. The circulant graphs with two chord lengths and recursive circulant graphs have been proven to be decomposable into hamiltonian cycles.

A graph is called a circulant graph (or simply a circulant) if it possesses all automorphism which cyclically permutes all the vertices. Circulants are vertex-transitive graphs with interesting and attractive properties.

From the earliest days of the study of circulants it became clear that their properties depend heavily on the multiplicative nature of its order (a graph is said to be of order $n$ if $n$ is the number of its vertices). The structural theory is simple for prime orders $n=p$ (though the divisors of $p-1$ should also be taken into account). Accordingly, it is a simple matter to count non-isomorphic $p$-circulants and this has been done by several researchers for various types of directed and undirected graphs. But every wider class of orders required a great amount of efforts. A considerable progress has been achieved in studying circulants for prime-power and (more recently) square-free orders. Here we are concerned with both these cases.If some combinatorial objects are natural and interesting for study, their enumeration (in various senses: constructive, analytical and asymptotic) is also a natural and interesting task. This is the case for circulants. As typical for enumerative combinatorics in general, only a certain small but highly rigorous part of structural and algebraic properties of objects is significant for counting. In principle, the isomorphism theorems that have been previously obtained for the above-mentioned orders $n$ are sufficient to count circulants analytically. However the real picture is somewhat more vague.First of all, by their nature, circulants are not only combinatorial but algebraic and number-theoretic objects as well. Therefore they are described in terms slightly inconvenient for enumerative combinatorics. On the other hand, the base group "up to which" circulants are counted looks very simple: it is a cyclic group or a direct product of cyclic groups. This facilitates the counting indeed and provides a systematic way for enumerating circulants of square-free orders. However, for the case of prime-power orders, another difficult problem arises instead (as we shall see later): a subtle and awkwardly structured set on which this group acts. Thus, there is a gap between the effective main algebraic isomorphism theorem for circulants of order $p^{k}$ and enumerative consequences from it.

A circular graph of order $n$ has the vertex set $V(G)=Z_{n}$ and edge set 


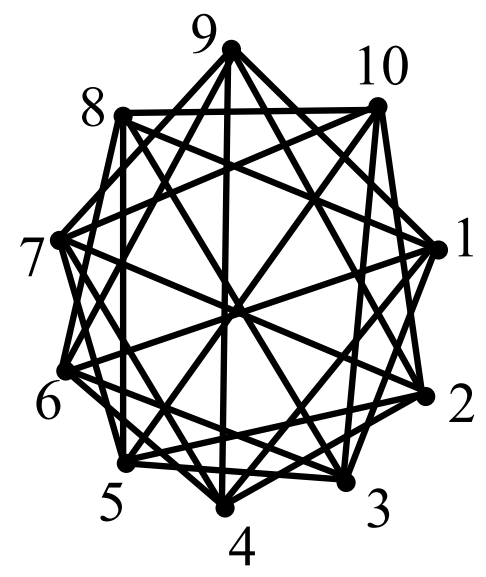

Figure 1: Circulant Graph $C_{10,\{2,3,5\}}$

$E(G)=\{u v: u-v \in D\}$ for some generating set $D \subseteq V(G)$. This set $D$ must not contain the identity element 0 , and must be closed under additive inverses. We say that $C_{n, D}$ is the circulant graph of order $n$ with generating set $D$. Note that $C_{n, D}$ is an undirected Cayley graph for the graph $G=\left(Z_{n},+\right)$ [28]. Thus, they are a special case of the more general family of Cayley graphs. An equivalent definitions of $C_{n, D}$ is: Given a set $D \subseteq\left\{1,2,3, \ldots,\left\lfloor\frac{n}{2}\right\rfloor\right\}$, the circulant graph $C_{n, D}$ is the graph with vertex set $V(G)=Z_{n}$ and edge set $E(G)=\left\{u v:|u-v|_{n} \in D\right\}$, where $|x|_{n}=\min \{|x|, n-|x|\}$ is the circular distance modulo $n$. For example, $C_{10,\{2,3,5\}}$ is shown in Figure 1.

Note that the circulant $C_{n,\left\{1,2,3, \ldots,\left\lfloor\frac{n}{2}\right\rfloor\right\}}$ is the complete graph $K_{n}$. Also $C_{n,\{1\}}$ is the cycle $C_{n}$. Further $C_{n,\{d\}}$ is the graph $C_{n}$ for any $d$ with $\operatorname{gcd}(d, n)=1$. For geometers, circulant graphs are known as star polygons [8]. Circulant graphs have been used to solve problems in graph theory [1] as well as number theory and analysis [21]. They are well studied in network theory as they model practical data connection networks [3], [33]. They have important applications to the theory of designs and error correcting codes [47].

\section{Circulant Graphs in the Study of Distance Graphs}

Distance graphs generalize the very well-studied class of circulant graphs. In fact, circulant graphs coincide exactly with the regular distance graphs [43]. In [14], [15], [43], [44], [45], some fundamental results concerning circulant graphs 
are extended to the more general class of distance graphs.

In a way, we can regard the integer distance graph $G(Z, D)$ as the infinite analogue of the circulant $C_{n, D}$.

Besides the chromatic number, other coloring parameters of distance graphs such as the fractional chromatic number and the circular chromatic number provide more information on the structure of distance graphs and are useful to determine the chromatic number of distance graphs. Further these coloring parameters of distance graphs are found closely related to some problems studied in number theory and geometry.

Let $r, x, y$ be reals with $0 \leq x, y<r$. The circular difference modular $r$ for $x$ and $y$, denoted as $|x-y|_{r}$, is defined as $|x-y|_{r}=\min \{|x-y|, r-|x-y|\}$. Let $k, d$ be positive integers with $k \geq 2 d$. A $(k, d)$-coloring of graph $G$ is a mapping $c: V(G) \rightarrow\{0,1, \ldots, k-1\}$, such that $|c(x)-c(y)|_{k} \geq d$ for any $x y \in E(G)$. The circular chromatic number $\chi_{c}(G)$ is the minimum ratio $(k / d)$ among all $(k, d)$-colorings of $G$.

A graph homomorphism from a graph $G$ to a graph $H$ is an edge preserving function from $V(G)$ to $V(H)$. If such a function exists, we say $G$ is homomorphic to $H$ and denote this by $G \rightarrow H$. The chromatic number of $G$ is the minimum $n$ such that $G \rightarrow K_{n}$. For positive integers $p, q$ with $p \geq 2 q$, the circular clique $K_{p / q}$ has as the vertex set $Z_{p}=\{0,1, \ldots, p-1\}$ where two vertices $u$ and $v$ are adjacent if $|u-v|_{p} \geq q$. The circular chromatic number of $G$ is the minimum ratio $p / q$ such that $G \rightarrow K_{p / q}$. From this point of view, $K_{p / q}$ plays essentially the same role as the one that $K_{n}$ does in vertex-coloring.

For a positive integer $n$, and a subset $D$ of $[n]=\{1,2, \ldots, n\}$, the $n$-vertex circulant graph generated by $D$, denoted by $G\left(Z_{n}, D\right)$, has as the vertex set $Z_{n}=\{0,1, \ldots, n-1\}$ and vertices $a$ and $b$ are adjacent if $|a-b|_{n} \in D$. The circular clique $K_{p / q}$ is a circulant graph, $K_{p / q}=G\left(Z_{p},[q-1]\right)$.

For any $D, G(Z, D) \rightarrow G\left(Z_{n}, D\right)$ holds for all $n \geq 2 \max D$. Hence a coloring parameter of the circulant graph $G\left(Z_{n}, D\right)$ provides an upper bound for $G(Z, D)$. For a lower bound, one may consider the subgraph of $G(Z, D)$ induced by the vertex set $\{0,1,2, \ldots, n-1\}$. (Note, this subgraph may not be isomorphic to $G\left(Z_{n}, D\right)$ ). The coloring parameters of this subgraph provides a lower bound for $G(Z, D)$.

The circular chromatic number of distance graphs is closely related to the parameter involved in the Wills conjecture [53]. The parameter $\kappa(D)$ is studied in the Diophantine approximations by Wills [53]. A long-standing open question concerning $\kappa(D)$ is the conjecture of Wills [53]: 'For any finite set $D$ of positive integers, $\kappa(D) \geq 1 /(|D|+1)$.' This conjecture has been given the name 'lonely runner conjecture' by Goddyn. Suppose $m$ runners run laps on a circular track 
of unit length. Each runner maintains a constant speed, and the speeds of all the runners are distinct. A runner is called lonely if the distance on the circular track between him or her and every other runner is at least $1 / \mathrm{m}$. Equivalently, the conjecture asserts that for each runner, there is a time when he or she is lonely. It is known that [62] for any set $D, \chi_{c}(Z, D) \leq 1 / \kappa(D)$ and $\max \left\{\omega(G), \frac{|V(G)|}{\alpha(G)}\right\} \leq \chi_{c}(G) \leq \chi(G),\left\lceil\chi_{c}(G)\right\rceil=\chi(G)$, where $\omega(G)$ is the clique size of $G$ and $\alpha(G)$ is the independence number of $G$. So $\omega(Z, D) \leq \chi_{c}(Z, D) \leq$ $1 / \kappa(D)$.

A discrete version of $\kappa(D)$ for finite sets $D$ is given as follows. For a set $D$, let $\lambda, p \leq 2 \max D$ be positive integers, with $\operatorname{gcd}(\lambda, p)=1$. Denote $(\lambda D)_{p}=\min \left\{|\lambda d|_{p}: d \in D\right\}$. The relation between $\chi_{c}(Z, D)$ and $k(D)$ can be established by the following method which also provides an upper bound for $\chi_{c}\left(Z_{n}, D\right)$. Suppose $\kappa(D)=q / p$. Then, there exists $p, \lambda \leq 2 \max D, \operatorname{gcd}(\lambda, p)=$ 1 such that $(\lambda D)_{p}=q$. The permutation $\Pi$ on $Z_{p}$ defined by $\Pi(x)=\lambda x$ is indeed a homomorphism from $G\left(Z_{p}, D\right)$ to the circular clique $K_{p / q}$. Therefore $\chi_{c}(Z, D) \leq \chi_{c}\left(Z_{p}, D\right) \leq p / q=1 / \kappa(D)$. The permutation $\Pi$ is called the multiplier method which has been used in several research articles on coloring parameters for circulant graphs. The multiplier method provides an upper bound for the circular chromatic number of distance graph and often the bound is sharp enough to determine the chromatic number. On the other hand, the multiplier method does not always provide the exact value for $\chi_{c}(Z, D)$, a particular case is when $\chi_{c}(Z, D)<\kappa(D)$.

The following result gives a useful upper bound for the chromatic number of a distance graph in terms of the chromatic number of a circulant graph [35], [46].

Theorem 5.1. Let $C_{n, D}$ be a circulant, where $D \subseteq\left\{1,2, \ldots,\left\lfloor\frac{n}{2}\right\rfloor\right\}$. Then, $\chi(Z, D) \leq \chi\left(C_{n, D}\right)$.

Proof. Define $D^{\prime}=D \cup\{n-x: x \in D\}$. Since $D \subseteq D^{\prime}$ and $\chi$ is a monotone it follows that $\chi(Z, D) \leq \chi\left(Z, D^{\prime}\right)$, as $|u-v| \notin D^{\prime}$ implies that $|u-v| \notin D$. In other words, any $k$-coloring of $G\left(Z, D^{\prime}\right)$ must also be a $k$-coloring of $G(Z, D)$.We now claim that $\chi\left(Z, D^{\prime}\right)=\chi\left(C_{n, D}\right)$. Then this Combined with the inequality $\chi(Z, D) \leq \chi\left(Z, D^{\prime}\right)$ will complete the proof. By definition, any proper coloring of $G\left(Z, D^{\prime}\right)$ must satisfy $u-v \notin D^{\prime}$ whenever $u>v$.

By the definition of $D^{\prime}$, any proper coloring of $C_{n, D}$ must satisfy $u-v \notin D^{\prime}$ whenever $u>v$. The condition for a proper coloring is identical for both graphs: the only difference is that $G\left(Z, D^{\prime}\right)$ is an infinite graph, while $C_{n, D}$ is not. We now justify that $\chi\left(Z, D^{\prime}\right)=\chi\left(C_{n, D}\right)$. First note that any $k$-coloring of $G\left(Z, D^{\prime}\right)$ can be made into a $k$-coloring of $C_{n, D}$ by taking its restriction to just 
the $n$ vertices of the circulant. Now to establish the converse: start with any $k$-coloring of $C_{n, D}$. This generates a $k$-coloring of $G\left(Z, D^{\prime}\right)$. For a particular $k$-coloring of $C_{n, D}$, let $\left\{V_{1}, V_{2}, \ldots, V_{k}\right\}$ be the set of color classes produced. We define the following tiling of the $k$ color classes: $T_{i}=V_{i}+n Z=\{x+n y$ : $x \in V_{i}, y \in Z$. $\}$. We now justify that each $T_{i}$ is independent in $G\left(Z, D^{\prime}\right)$, thus proving that the set $\left\{T_{1}, T_{2}, \ldots, T_{k}\right\}$ represents the color classes corresponding to a proper $k$-coloring of $G\left(Z, D^{\prime}\right)$. Suppose on the contrary that $a$ and $b$ are not independent in some $T_{i}$, where $a>b$. Then $|a-b|=a-b=p n+v_{1}-v_{2} \in D^{\prime}$, where $v_{1}, v_{2} \in V_{i}$ and $p$ is some non-negative integer. Since $0 \leq v_{1}, v_{2} \leq n-1$ and $1 \leq p n+v_{1}-v_{2} \leq n-1$, it follows that $p=0$ or $p=1$.If $p=0$, then $v_{1}-v_{2} \in D^{\prime}$, from above. So $v_{1}>v_{2}$. By definition of $D^{\prime}$, this implies that $v_{1}-v_{2} \in D$ or $n-\left(v_{1}-v_{2}\right) \in D$. In other words, $v_{1}$ and $v_{2}$ are not independent in $C_{n, D}$, and thus cannot belong to the same color class $V_{i}$. We have a contradiction. If $p=1$, then $n+v_{1}-v_{2} \in D^{\prime}$. So $v_{2}>v_{1}$. By definition of $D^{\prime}$, this implies that $n+v_{1}-v_{2}=n-\left(v_{2}-v_{1}\right) \in D$ or $n-\left(n+v_{1}-v_{2}\right)=v_{2}-v_{1} \in D$. In other words, $v_{1}$ and $v_{2}$ are not independent in $C_{n, D}$, and thus cannot belong to the same color class $V_{i}$. We have a contradiction. This establishes the converse, that every $k$-coloring of $C_{n, D}$ can be extended to a $k$-coloring of $G\left(Z, D^{\prime}\right)$. Hence we conclude that $\chi\left(Z, D^{\prime}\right)=\chi\left(C_{n, D}\right)$. Since we have already proven that $\chi(Z, D) \leq \chi\left(Z, D^{\prime}\right)$, the proof is complete.

There are infinitely many sets $D$ for which equality does not hold. As a simple example, consider the case $n=4$ and $D=\{1,2\}$. Then $\chi(Z, D)=3$, while $\chi\left(C_{n, D}\right)=\chi\left(K_{4}\right)=4$.

The existence of a minimum coloring of an integer distance graph $G(Z, D)$ with finite distance set $D$ implies the existence of a periodic minimum coloring. A coloring $f: Z \rightarrow\left\{c_{1}, \ldots, c_{k}\right\}$ is called periodic with period $p$ if $f(v)=f(v+$ $p) ; p \in N$. To see this, partition the integer line into segments of length $y+$ $1, y=\max \{x: x \in D\}$ and assume that $G(Z, D)$ is properly $k$-colored by some function $f$. Since there are finitely many different ways to color a single element (namely $k^{y+1}$ different patterns) and the integer line has an infinite number of segments, it follows that there exists two segments $\left[v_{0}, v_{y}\right]$ and $\left[w_{0}, w_{y}\right], w_{0}>\neq$ $v_{0}$ colored with the same coloring pattern by function $f$. Then $G(Z, D)$ can be properly re-colored with $k$ colors with the pattern defined by segment $\left[v_{0}, w_{0}-1\right]$ and period $p=w_{0}-v_{0}$.

In order to show the relationship between integer distance graphs and circulant graphs, consider a finite set $D$. Let $n \in Z^{+}$and let $D=\left\{d_{1}, d_{2}, \ldots, d_{s}\right\}$ with $D \subseteq\{1,2, \ldots,\lfloor n / 2\rfloor\}$. The circulant graph can be thought of as a graph $G_{c}(n, D)$ of order $n$ with vertex set $V=\{0,1, \ldots, n-1\}$ with an edge joining $i$ and $j$ whenever $i=\left(j+d_{s}\right)(\bmod n), d_{s} \in D$. The existence of $\chi(G(Z, D))$ with 
period $p$ implies $\chi\left(G_{c}(y p, D)\right)=\chi(G(Z, D)), y \in Z^{+}$. However, $\chi\left(G_{c}(n, D)\right)$ with $y p+1 \leq n<(y+1) p$ remains specific to circulant graphs. For example, if $D=\left\{d_{1}, d_{2}, \ldots, d_{s}\right\}$ be a finite set of positive odd integers, then $G(Z, D)$ is a bipartite graph with vertex sets $V_{1}$ and $V_{2}$, containing odd and even integers respectively. So $\chi(G(Z, D))=2$ with period $p=2$ implies $\chi\left(G_{c}(n, D)\right)=2$ for any even $n$. But it is easy to see that $\chi\left(G_{c}(n, D)\right) \geq 3$ for $n$ odd. For, let $G(V, E)$ be a simple $k$-regular graph with $1 \leq k \leq|V|-1$. If the order of $G$ is odd, then $\chi(G) \geq 3$. Suppose not. Then $G$ is 2-colorable and hence there exists vertex partitions $V_{1}$ and $V_{2}$ where $V_{1} \cap V_{2}=\phi$ and $\left|V_{1}\right|+\left|V_{2}\right|=|V(G)|=2 l+1$, for some $l \in Z^{+}$. Now there are $k\left|V_{i}\right|$ edges leaving partition $V_{i}, i=1,2$. But the number of edges entering and leaving each partition has to be equal and this implies that $k\left|V_{1}\right|=k\left|V_{2}\right|$. This however is a contradiction to the odd order of $G$. In view of this, we consider $G_{c}(n, D)$, with $D \neq \phi$ and $n=2 l+1, l \in Z^{+}$, then as $G$ is a regular graph of degree greater than or equal to one the result follows. In general, for an arbitrary distance set $D$, the chromatic number of $G(Z, D)$ gives a lower bound for $\chi\left(G_{c}(n, D)\right)$. One can see [36] for more on circulant graphs.

\section{Pseudochromatic Coloring, Circulant Graphs and Distance Graphs}

A Pseudochromatic $k$-coloring of a graph $G$ is an assignment of at most $k$ colors to the vertices of $G$ such that two vertices with a common neighbor must receive different colors. The pseudochromatic number $\psi_{c}(G)$ of a graph $G$ is the minimum $k$ such that $G$ has a pseudochromatic $k$-coloring. Note that a peudochromatic coloring need not be a proper coloring. That is, here the adjacent vertices can have the same color.

The distance 2 graph (also called square graph) denoted $G^{2}$ of a graph $G(V, E)$ is the graph on the vertex set $V$ in which two vertices are joined by an edge if their edge distance in $G$ is at most 2.

Theorem 6.1. For a graph $G$, we have $\chi\left(G^{2}\right) \leq 2 \psi_{c}(G)$.

Proof. Let $m=\psi_{c}(G)$. Then there exists a partition $V_{1}, V_{2}, \ldots, V_{m}$ of the vertex set $V(G)$ of $G$ such that no two vertices in $V_{i}$ have a common neighbor in $G$. Then the set of edges with both ends in $V_{i}$ induces a matching. Therefore we can partition $V_{i}$ into two sets $U_{i}$ and $W_{i}$ both independent in $G$. Then $U_{i}$ and $W_{i}$ are independent in $G^{2}$ also, as no two vertices in $U_{i}$ or $W_{i}$ have a common neighbor in $G$. This implies $U_{1}, \ldots, U_{m}, W_{1}, \ldots, W_{m}$ forms a partition of $G^{2}$ into independent sets in $G^{2}$. Therefore $\chi\left(G^{2}\right) \leq 2 m$. 
Now consider a circulant graph $G_{c}$ on a set $V=\{0,1, \ldots, 3 n-1\}$ such that two vertices $u$ and $v$ are adjacent if and only if $u-v \equiv \pm 1(\bmod 3 n)$, where $n$ is even. Then it is easy to see that $G_{c}^{2}=K_{3 n}$ and hence $\chi\left(G_{c}^{2}\right)=3 n$. By the above theorem, $\psi_{c}\left(G_{c}\right) \geq 3 n / 2$. If $u, v$ are the vertices with $u-v \equiv \pm 1(\bmod$ $3 n$ ), then they will have no common neighbors. Therefore in a pseudochromatic coloring, we can color pairs of consecutive integers with the same color and so $\psi_{c}\left(G_{c}\right) \leq\lceil 3 n / 2\rceil$. As $n$ is even, we get that $\psi_{c}\left(G_{c}\right)=3 n / 2$.

\section{Conclusion}

We have ascertained with a number of instances that the study of chromatic number of circulant graphs have pertinent role in the study of chromatic number of distance graphs. We conclude with a few open problems.

1. Find the minimum $n_{0}$ such that for all $n \geq n_{0}$, the circulant graph of order $n$ and generating set $D=\left\{d_{1}, d_{2}\right\}$ has minimum chromaticity.

2. Given a cardinality $k$, find sets $D$ with $|D|=k$ such that $\chi\left(G_{c}(n, D)\right) \leq 3$.

3. For an arbitrary set $D$, determine whether there exists a value $x$ such that for all $n \geq n_{0} \chi\left(G_{c}(n, D)\right) \leq x$.

\section{Acknowledgments}

This research is carried out with the financial grant and support of National Board for Higher Mathematics, Government of India under the grant sanction No. 2/48(10)/2010/R\&D-II/11192/dated 26, Nov, 2010.

\section{References}

[1] B. Alspach, T. Parsons, Isomorphism of circulant graphs and digraphs, Disc. Maths., 25, No. 2 (1979), 97-108. doi: 10.1016/0012-365X(79)900116.

[2] B. Green, T. Tao, The Primes contain arbitrarily long arithmetic progressions, Annals of Mathematics, 167, No. 2 (2008), 481-547. doi: 10.4007/annals.2008.167.481. 
[3] J.C. Bermond, F. Comellos, D.F. Hsu, Distributed loop computer networks: a survey, Journal of Parallel and Distributed Computing, 24, No. 1 (1985), 2-10. doi: 10.1006/jpdc.1995.1002.

[4] W. Bienia, L. Goddyn, P. Gvozdjak, A. Sebo, M. Tarsi, Flows, view obstructions, and the lonely runner, J. Combin. Theory (B), 72, No. 1 (1998), 1-9. doi: $10.1006 /$ jctb.1997.1770.

[5] D.G. Cantor, B. Gordon, Sequences of integers with missing differences, J. Combin. Theory Ser. A, 14, No. 3 (1973) 281-287. doi: 10.1016/00973165(73)90003-4.

[6] G.J. Chang, L. Huang, X. Zhu, The circular chromatic number and fractional chromatic number of distance graphs, European Journal of Combinatorics, 19, No. 4 (1998), 423-431. doi: 10.1006/eujc.1997.0199.

[7] G.J. Chang, D. Liu, X. Zhu, Distance graphs and T-coloring, J. of Comb. Theory Ser B, 75 (1999), 159-169.

[8] G.J. Chang, L. Huang, Circular chromatic number of distance graphs with distance sets missing multiples, European J. of Combinatorics, 21, No. 2 (2000), 241-248. doi: 10.1006/eujc.1999.0284.

[9] J.J. Chen, G.J. Chang, K.C. Huang, Integral distance graphs, J. of Graph Theory, 25, No. 4 (1997), 287-294. doi: 10.1002/(SICI)10970118(199708)25:4.

[10] Y.G. Chen, On a conjecture about Diophantine approximations, II, Number Theory, 37, No. 2 (1991), 181-198. doi: 10.1016/S0022-314X(05)800368.

[11] Y.G. Chen, On a conjecture about Diophantine approximations, III, Number Theory, 39, No. 1 (1991), 91-103. doi: 10.1016/0022-314X(91)90036-B.

[12] Y.G. Chen, On a conjecture about Diophantine approximations, IV, Number Theory, 43, No. 2 (1993), 186-197. doi: 10.1006/jnth.1993.1016.

[13] K.B. Chilakamarri, The unit-distance graph problem: a brief survey and some new results, Bulletin of the Institute of Combinatorics and its Applications, 8(1993), 39-60.

[14] M. Chin Lin, D. Rautenbach, F.J. Soulignac, J.L. Szwarcfiter, Powers of cycles, powers of paths, and distance graphs, Discrete Appl. Maths., 159, No. 7 (2011), 621-627. doi: 10.1016/j.dam.2010.03.012. 
[15] C. Lowenstein, D. Rautenbach and F. Regen, On Hamiltonian paths in distance graphs, Applied Mathematics Letters, 24, No. 7 (2011), 1075-1079. doi: $10.1016 /$ j.aml.2011.01.025.

[16] H.S.M. Coxeter, Twelve Geometric Essays, Southern Illinois University Press, Carbondale/EdwardsVille, IL (1968).

[17] T.W. Cusick, View-obstruction problems, Aequationes Math., 9, No. 2-3 (1973), 165-170. doi: 10.1007/BF01832623.

[18] T.W. Cusick, View-obstruction problems II, Proc. Amer. Math. Society, 84 (1982), 25-28.

[19] T.W. Cusick, View-obstruction problems in $n$-dimensional geometry, $J$. Combin. Theory (A), 16 (1974), 1-11.

[20] T.W. Cusick, C. Pomerance, View-obstruction problems, III, J. Number Theory, 19 (1984), 131-139. doi: 10.1016/0022-314X(84)90097-0.

[21] P.J. Davis, Circulant Matrices, 2nd edition, Chelsea Publishing, NewYork (1994).

[22] R.B. Eggleton, New results on 3-chromatic prime distance graphs, Ars Combin., 26B (1988), 153-180.

[23] R.B. Eggleton, P. Erdos, D.K. Skilton, Update information on research problem 77, Dis. Math., 69, No. 1 (1988), 105. doi: 10.1016/0012365X(88)90184-7.

[24] R.B. Eggleton, P. Erdos, D.K. Skilton, Research problem 77, Dis. Math., 58, No. 3 (1986), 323. doi: 10.1016/0012-365X(86)90153-6.

[25] R.B. Eggleton, P. Erdos, D.K. Skilton, Coloring the real line, J. Combinatorial Theory Ser B, 39 (1986), 86-100 to erratum 41, No. 1 (1986), 139. doi: 10.1016/0095-8956(85)90039-5.

[26] R.B. Eggleton, P. Erdos, D.K. Skilton, Coloring prime distance graphs, Graphs and Combinatorics, 6, No. 1 (1990), 17-32.

[27] R. Elliger, Uber ein problem der verkehrsoptimierung und eir.e von P. Erdos et al. geauberte vermutung, Ph.D. Thesis, Institut fur Mathematics, Technische Hochschule, Illmenav, Germany (1988). 
[28] C.D. Godsil, Algebraic Combinatorics, Chapman and Hall, New York (1993).

[29] J. Griggs, D. Liu, Channel assignment problem for mutually adjacent sites, J. Combinatorial Theory Series A, 68, No. 1 (1994), 169 -183. doi: 10.1016/0097-3165(94)90096-5.

[30] N.M. Haralambis, Sets of integers with missing differences, J. Combin. Theory (A), 23, No. 1 (1977), 22-33. doi: 10.1016/0097-3165(77)90076-0.

[31] G.H. Hardy, J.E. Littlewood, Some problems of partitio numerorum: III: on the expression of a number as a sum of primes, Acta Math., 44 (1923), $1-70$.

[32] D.R. Heath-Brown, Three primes and an almost-prime in arithmetic progression, J. London math. Soc., 23, No. 3 (1981), 396-414. doi: 10.1112/jlms/s2-23.3.396.

[33] F.K. Hwang, A survey on multi-Loop Networks, Theoretical Computer Science, 219 (2003), 107-121. doi: 10.1016/S0304-3975(01)00341-3.

[34] L.L. Ivanov, On the chromatic numbers of $R^{2}$ and $R^{3}$ with intervals of forbidden distances, Electronic Notes in Discrete Mathematics, 29 (2007), 159-162.

[35] J. Brown, R. Hoshino, Proof of a conjecture on fractional ramsey numbers, Journal of Graph Theory (2009), 163-178 (www.interscience.wiley.com). doi: $10.1002 /$ jgt.20416.

[36] J. Barajas, O. Serra, On the chromatic number of circulant graphs, Discrete Mathematics, 309, No. 18 (2009), 5687-5696. doi: 10.1016/j.disc.2008.04.041.

[37] A. Kemnitz, H. Kolberg, Coloring of integer distance graphs, Discrete mathematics, 193 (1996), 13-123.

[38] A. Kemnitz, M. Marangio, Chromatic number of integer distance graphs, Discrete mathematics, 233, No. 1-3 (2001), 239-246. doi: 10.1016/S0012365X(00)00243-0.

[39] A. Kemnitz, M. Marangio, Edge colorings and total colorings of integer distance graphs, Discussiones mathematicae, 22, No. 1 (2002), 149-158. doi: $10.7151 /$ dmgt.1164. 
[40] A. Kemnitz, M. Marangio, Colorings and list colorings of integer distance graphs, Congressus Numerantium, 151 (2001), 75-84.

[41] W. Lin, Some star extremal circulant graphs, Discrete Mathematics, 271, No.1-3 (2003), 169-177. doi: 10.1016/S0012-365X(02)00872-5.

[42] D.D-F. Liu, X. Zhu, Distance graphs with missing multiples in the distance sets, J. of Graph Theory, 30, No. 4 (1999), 245-259. doi: 10.1002/(SICI)1097-0118(199904)30.

[43] L.D. Penso, D. Rautenbach, J.L. Szwarcfiter, Cycles, paths, connectivity and diameter in distance graphs, in: Graph-Theoretic Concepts in Computer Science, 35th International Workshop, WG 2009, Montpellier France, June 2009, LNCS, 5911 (2010), 320-328.

[44] L.D. Penso, D. Rautenbach, J.L. Szwarcfiter, Connectivity and diameter in distance graphs, Networks, 57, No. 4 (2011), 310-315. doi: $10.1002 /$ net.20397.

[45] L.D. Penso, D. Rautenbach, J.L. Szwarcfiter, Long cycles and paths in distance graphs, Discrete math., 310, No. 23 (2010), 3417-3420. doi: 10.1016/j.disc.2010.07.020.

[46] Richard Hoshino, Independence polynomials of circulant graphs, Doctoral Thesis, Dalhousie University, October 15 (2007).

[47] V. Sachkov, V.E. Tarakanov, Combinatorics of Nonnegative matrices, Translations of Mathematical Monographs, 213 AMS, Providence (2002).

[48] J.G. Van Der Corput, Uber Summen von primzahlen und primzahlquadraten, Math Ann., 116 (1939), 1-50.

[49] M. Voigt, Coloring distance graphs, Ars Combinatoria, 52 (1999), 3-12.

[50] M. Voigt, H. Walther, Chromatic number of prime distance graphs, Discrete App. Math, 51, No. 1-2 (1994), 197-209. doi: 10.1016/0166218X(94)90109-0.

[51] M. Voigt, H. Walther, On the chromatic number of special distance graphs, Discrete Math., 97, No. 1-3 (1991), 395-397. doi: 10.1016/0012365X(91)90454-A.

[52] H. Walther, Uber eine spezielle klasse unendlicher graphen in: graphentheorie II (Wissenschaftsverlag), Mannheim (1990), 268-295. 
[53] J.M. Wills, ZweiSatze Uber inhomogene diophantische approximation von irrationlzahlen, Monatsch. Math., 71 (1967), 263-269.

[54] V. Yegnanarayanan, Chromatic number of graphs with special distance sets, I, Algebra and Discrete Mathematics, Accepted for publication (2013).

[55] V. Yegnanarayanan, On a question concerning prime distance graphs, Discrete Math., 245, No. 1-3 (2002), 293-298. doi: 10.1016/S0012365X(01)00221-7.

[56] V. Yegnanarayanan, A. Parthiban, Chromatic number of certain graphs, Proc. of International Conference on Mathematics in Engineering and Business Management, Stella Maris College, Chennai, I, No. 1 (2012), 115-118 (ISBN 978-81-8286-015-5).

[57] V. Yegnanarayanan, A. Parthiban, Chromatic number of graphs with special distance sets-II, Proc of International Conference on Mathematical Modeling and Applied Soft Computing, CIT, Coimbatore, India, I, No. 1 (2012), 305-313 (ISBN 978-81-923752-1-2).

[58] V. Yegnanarayanan, A. Parthiban, Chromatic number of graphs with special distance sets-III, Journal of Mathematical and Computational Sciences, 2, No. 5 (2012), 1257-1268.

[59] V. Yegnanarayanan, The chromatic number of generalized Fibonacci prime distance graph, Journal of Mathematical and Computational Sciences, 2, No. 5 (2012), 1451-1463.

[60] V. Yegnanarayanan, A. Parthiban, Chromatic number of graphs with special distance sets-IV, Proc of International Conference on Applied Mathematics and Theoretical Computer Science(ICAMTCS), 1, No. 1 (2013), 71-76 (ISBN 978-93-82338-30-7).

[61] V. Yegnanarayanan, A. Parthiban, Chromatic number of graphs with special distance sets-V, Open Journal of Discrete Mathematics, 3, No. 1 (2013), 1-6. doi: 10.4236/ojdm.2013.31001.

[62] X. Zhu, Circular chromatic number: a survey, Dis. Maths., 229, No. 1-3 (2001), 371-410. doi: 10.1016/S0012-365X(00)00217-X.

[63] X. Zhu, Pattern periodic coloring of distance graphs, J. Comb Theory Ser $B, 73$ (1997), 195-206. 
[64] X. Zhu, The Circular chromatic number of distance graphs with distance sets of cardinality 3, J. Graph Theory, 41, No. 3 (2002), 195-207. doi: 10.1002/jgt.10062.

[65] X. Zhu, The Circular chromatic number of a class of distance graphs, Discrete Math., 265, No. 1-3 (2003), 337-350. doi: 10.1016/S0012365X(02)00631-3. 